\title{
Scattering of Light from Assemblies of Truncated and Disordered Spherulites
}

\author{
Toshihide IsHIKAWA ${ }^{* 1}$ and Richard S. STEIN ${ }^{* 2}$ \\ ${ }^{* 1}$ Government Industrial Research Institute, Osaka, \\ Midorigaoka 1, Ikeda, Osaka, Japan. \\ *2Polymer Research Institute and Department of Chemistry, \\ University of Massachusetts, Amherst, \\ Massachusetts 01002, U.S.A.
}

(Received January 26, 1976)

\begin{abstract}
The light-scattering patterns from two-dimensional statistically arranged spherulites have been examined by using a combination model which takes into account the size distribution, truncation, and disorder effects. From the computer simulation for assemblies of truncated spherulites, it is found that the distribution of their average radii can be fit by a logarithmic Gaussian frequency function, but their scattering patterns, based upon the estimated distribution parameters, do not represent those for the assemblies of the same truncated spherulites. Refinement of the model used in the previous works is possible in order to make a more quantitative estimation of light-scattering intensity for semi-crystalline polymer films and to clarify the origin of excess $\mathrm{H}_{\mathrm{v}}$ scattering.

\section{KEY WORDS Light Scattering / Size Distribution / Truncation / \\ Disorder /}

Since the study of light scattering from crystalline polymer films was proposed by Stein and Rhodes, ${ }^{1}$ a number of papers $^{2}$ have been devoted to accounting for the deviation between theoretical and experimental light-scattering patterns. Comprehensive discussions have recently been made by Yoon and Stein. ${ }^{3}$ The principal observations are that the experimental $H_{v}$ scattered pattern is found to become broader at small and at large scattering angles than the width given by the theory of a single spherulite, ${ }^{1}$ and higher order scattering maxima are not observed. Attempts to interpret the above observations by using the model approach in the recent studies of Stein and coworkers are based upon considerations of size distribution, ${ }^{4}$ truncation, ${ }^{5,6}$ and disorder effects. ${ }^{3,7}$ However, the separation of these effects and the simplification of the model leads to a shortcoming in the qualitative analysis of light-scattering patterns for semi-crystalline materials.

This paper differs from the statistical approach $^{8,9}$ in that the above comprehensive model effects are considered. That is, the scattering from an assembly of truncated spherulites is treated in a way which includes the distribution of spherulite sizes as well as the disorder in orientation of the optic axes within the lattice cells of a two-dimensional spherulite generated with a computer.

Although others, ${ }^{10}$ referring to the effect of size distribution, have stated that this does not give rise to any essential change in the scattered intensity, their conclusion seems to be somewhat premature, because of the reasons discussed in a later section.

\section{DEFINITION OF EQUATIONS}

The scattered amplitudes of $H_{\nabla}$ and $V_{v}$ scattering for a two-dimensional isolated spherulite when $\omega=0^{\circ}$ and $\beta=90^{\circ}$ (i.e., $\omega=0^{\circ}$ represents the optic axis lying in the plane of a spherulite and $\beta$ is the angle between the optic axis and the spherulite fibril direction and is $90^{\circ}$ for polyethylene) are given by ${ }^{11}$

$$
\begin{aligned}
E_{\mathrm{H}_{\mathrm{v}}}= & E_{0}\left(\alpha_{\mathrm{r}}-\alpha_{\mathrm{t}}\right) \cos \rho_{2} \sin 2 \mu\left(A / w^{2}\right) \\
& \times\left[2\left(1-J_{0}(w)\right)-w J_{1}(w)\right]
\end{aligned}
$$

and 


$$
\begin{aligned}
E_{\mathrm{V}_{\mathrm{v}}}= & E_{0} \cos \rho_{1}\left(2 A / w^{2}\right)\left[\left(\alpha_{\mathrm{r}}-\alpha_{\mathrm{s}}\right)\left(1-J_{0}(w)\right)\right. \\
& +\left(\alpha_{\mathrm{t}}-\alpha_{\mathrm{s}}\right)\left(w J_{1}(w)+J_{0}(w)-1\right) \\
& \left.+\left(\alpha_{\mathrm{t}}-\alpha_{\mathrm{r}}\right) \cos ^{2} \mu\left(2\left(1-J_{0}(w)\right)-w J_{1}(w)\right)\right]
\end{aligned}
$$

where $E_{0}$ is the physical constant, the polarizabilities $\alpha_{\mathrm{r}}$ and $\alpha_{\mathrm{t}}$ lie in the radial and the tangential direction of a spherulite, and $w$ equals $2 \pi R \sin \theta / \lambda ; R$ and $\lambda$ are the radius of a spherulite, and the wavelength in the medium, respectively. $J_{0}(w)$ and $J_{1}(w)$ are the zero- and firstorder Bessel functions. $\cos \rho_{1}$ and $\cos \rho_{2}$ are defined by ${ }^{11}$

$$
\begin{aligned}
& \cos \rho_{1}=\cos \theta /\left(\cos ^{2} \theta+\sin ^{2} \theta \cos ^{2} \mu\right)^{1 / 2} \\
& \cos \rho_{2}=\cos \theta /\left(\cos ^{2} \theta+\sin ^{2} \theta \sin ^{2} \mu\right)^{1 / 2}
\end{aligned}
$$

The averaged scattering intensity per unit area for the assembly of spherulites is given by

$$
I=(c / 4 \pi N) \sum E_{i}^{2} / \Sigma A_{i}
$$

where $N$ is the number of spherulites, $c$ is the velocity of light, $A_{i}$ is the area of individual spherulites, and the summation extends from 1 to $N$. An intensity function for the distribution of spherulite size, assuming noncorrelation between spherulites, may be expressed as: ${ }^{4}$

$$
I(R)=\int I(R) P(R) \mathrm{d} R / \int P(R) \mathrm{d} R
$$

Here $P(R)$ is the distribution function and is defined by a normalized Gaussian form ${ }^{4}$ and/or a normalized logarithmic Gaussian form ${ }^{12}$ as follows:

$$
P(R)=\frac{1}{(2 \pi)^{1 / 2} \sigma} \exp \left[-\frac{(R-\bar{R})^{2}}{2 \sigma^{2}}\right]
$$

or

$$
P(R)=\frac{\exp \left[-\left(\ln R-\ln R_{\mathrm{m}}\right)^{2} / 2 \sigma_{0}{ }^{2}\right]}{(2 \pi)^{1 / 2} \sigma_{0} R_{\mathrm{m}} \exp \left(\sigma_{0}{ }^{2} / 2\right)}
$$

where $\bar{R}$ and $\sigma$ are the mean radius of the spherulite and its standard deviation, and $R_{\mathrm{m}}$ and $\sigma_{0}$ represent the modal value of the radius of the spherulite and the width and skewness of the distribution, respectively. $\sigma_{0}$ is related to $\sigma$ by

$$
\sigma=R_{\mathrm{m}} \sigma_{0}, \quad \sigma_{0} \ll 1
$$

Kerker, et al.,${ }^{12}$ have recommended the use of eq 8 for the study of the size distribution of latex particles. Coherent interference of the scattering from different spherulites is neglected here.

\section{DISTRIBUTION AND TRUNCATION EFFECTS}

Previous studies ${ }^{3,4,10}$ were lacking in careful examination of the effect of size distribution parameters, for the reasons aforementioned. To find the population of distribution of spherulite sizes, it is necessary to establish random locations for an assembly of nuclei of spherulites in a given area by generation of random numbers with a computer. ${ }^{13}$ It is assumed here that the boundary line resulting from impingement of neighbor spherulites and the intersection line connected the two nuclei are perpendicular and the distances between the cross points at the boundary of the intersection line are equal. These assumptions may be limiting for real spherulitic polymers, since nuclei are known to propagate in three dimensions. It also involves the assumption of simultaneous activation of predetermined nuclei. Such an approximation, however, seems permissible for thin films from the observation of the photomicrograph of polyethylene under the normal conditions. ${ }^{6,14}$ The effect of the position of the nucleus in the spherulite will be discussed later.

A typical example of an assembly of truncated spherulites is illustrated in Figure 1, which represents 67 spherulites in a circle of $90 \mu \mathrm{m}$ diameter. The average radius $\langle\bar{R}\rangle$ is $4.9 \mu \mathrm{m}$

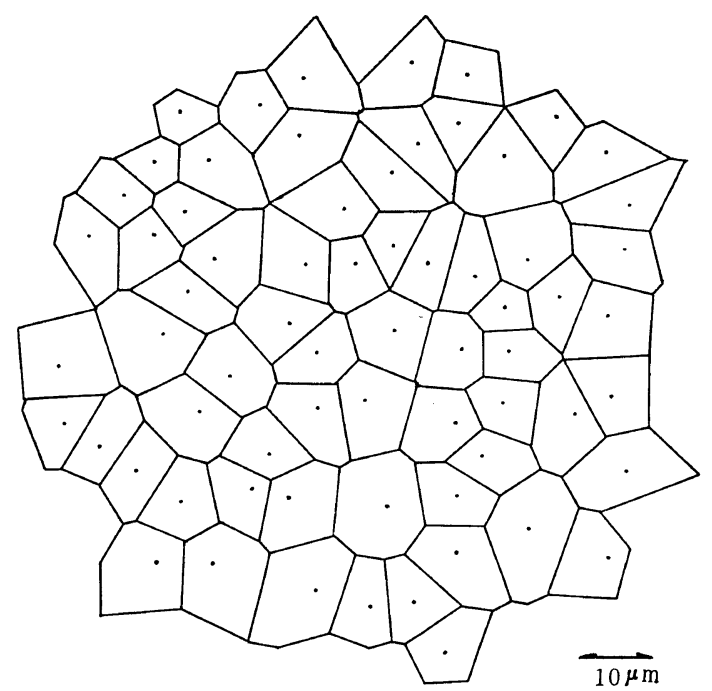

Figure 1. Schematic representation of an assembly of truncated spherulites generated by computer simulation. 
Light Scattering by Computer Simulation

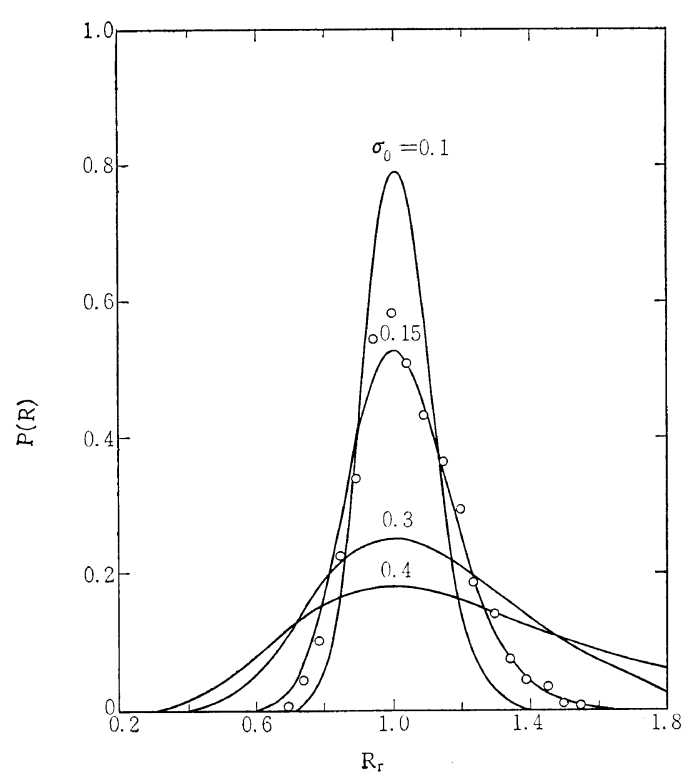

Figure 2. Frequency of logarithmic Gaussian distribution as functions of $\sigma_{0}$ and $R_{\mathrm{r}} . R_{\mathrm{r}}$ is the ratio of the spherulite radius to its modal value. Circles express the frequency for size distribution after averaging out the radii of truncated spherulites generated by a computer.

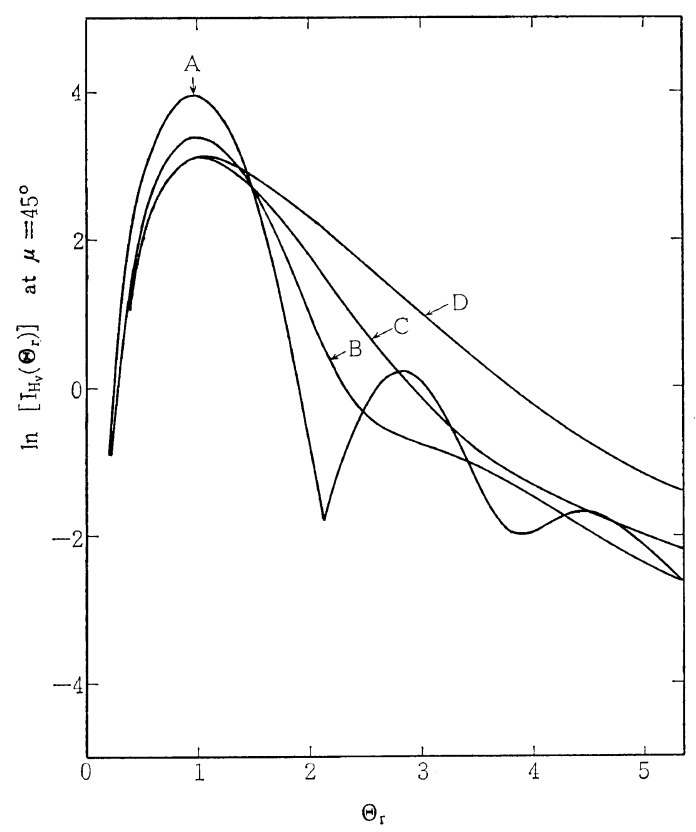

(a) and the average standard deviation of radius $\langle\sigma\rangle$ is $1.32 \mu \mathrm{m}$. Here dual averagings are made: the first is the average of the radius $(\bar{R})$ in asymmetric spherulites, and the variance is defined as an internal size distribution; the second makes an averaging for their mean radii in an assembly $(\langle\bar{R}\rangle)$, and this distribution is defined as an external one. A more exact distribution is obtained from 20 similar assemblies of spherulites (the total number of spherulites is 1225), which led to the result that $\langle\bar{R}\rangle=5.4 \mu \mathrm{m}$, and $\langle\sigma\rangle=1.45 \mu \mathrm{m}$, so that the truncation parameter is $\left\langle\sigma^{2} / \bar{R}^{2}\right\rangle=0.07$. The last value is in the range
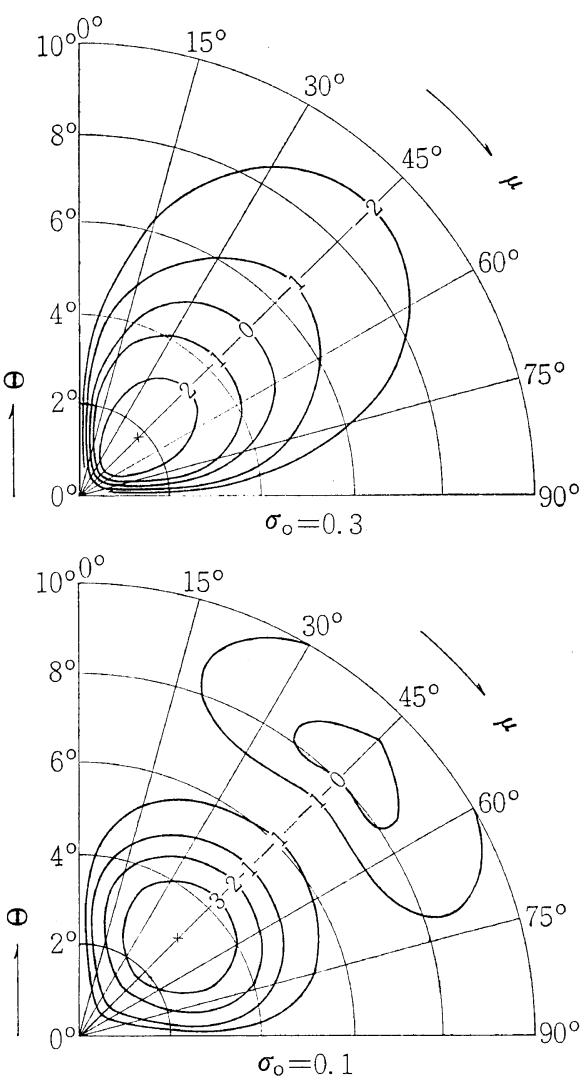

(b)

Figure 3. Effect of size distribution on the logarithmic $\mathrm{H}_{\nabla}$ scattering intensity for circular spherulites. a) Lines are plotted as a function of $\Theta_{\mathrm{r}}$, which is the ratio of scattering angle $\theta$ to $\theta_{\max }$ at maximum intensity and at $\mu=45^{\circ}: \mathrm{A}, 0.1 ; \mathrm{B}$, 0.2 ; C, $0.3 ; \mathrm{D}, 0.4$ for $\sigma_{0} ; R_{\mathrm{m}}=5 \mu \mathrm{m}$. b) One quadrant of intensity contours for $\sigma_{0}=0.1$ and 0.3 as functions of $\theta$ and $\mu$. 
of the experimental observation, $0.07-0.13 .^{6}$ Figure 2 depicts the frequency of the logarithmic Gaussian distribution with $R_{\mathrm{m}}=5 \mu \mathrm{m}$ for several values of $\sigma_{0}$. In addition, the circles in Figure 2 represent the frequency of distribution as a function of the average radii of the computergenerated 1225 truncated spherulites. Here $R_{\mathrm{r}}$ is the ratio of the radius to that of the modal value. It is seen that the line of $\sigma_{0}=0.15$ fits satisfactorily to the circles, except in the vicinity of the peak. Moore ${ }^{14}$ has briefly reported the distribution of spherulite sizes of crystalline polymers as studied by photomicrography and photographic light scattering. The width of $R_{\mathrm{r}}$ obtained with Marlex 6050 polyethylene was in the range of $0.71-1.41$. Similar values were obtained by Prud'homme ${ }^{6}$ from Marlex 50 poly-
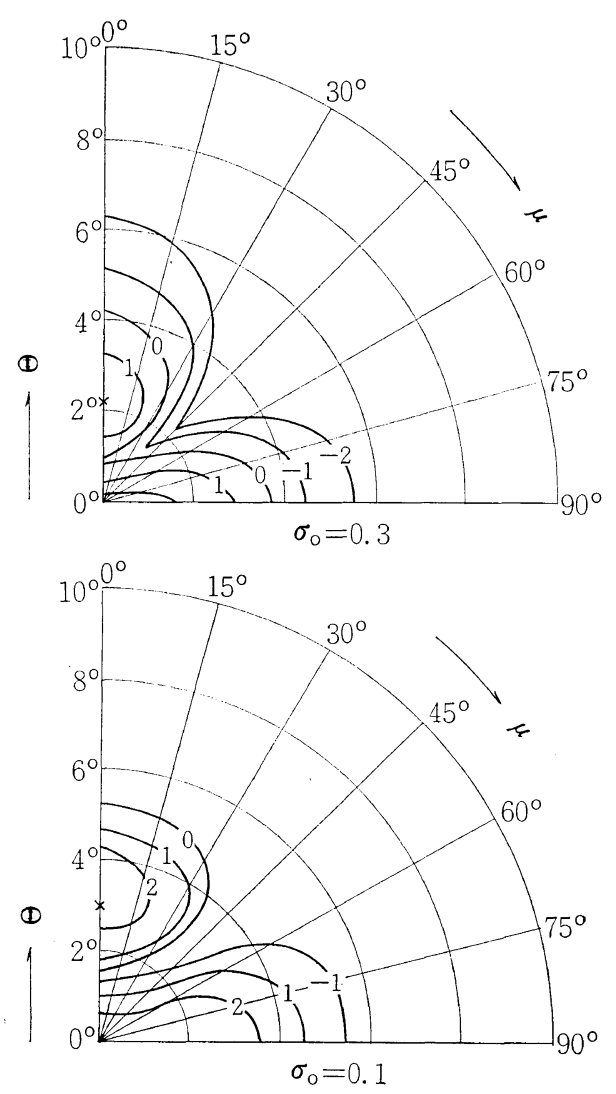

Figure 4. Effect of size distribution on the logarithmic $\mathrm{V}_{\mathrm{v}}$ scattering intensity for circular spherulites with $R_{\mathrm{m}}=5 \mu \mathrm{m}$ and $\alpha_{\mathrm{s}}=\left(\alpha_{\mathrm{r}}+2 \alpha_{\mathrm{t}}\right) / 3$ as functions of $\theta$ and $\mu$. ethylene by consideration of the truncation effect. Although from the above reports we are not able to estimate the frequency of size distribution, the width is in rather good accord with that from our computer simulation.

Let us examine the effect of the size distribution of non-truncated spherulites. In Figure 3a, the logarithmic $\mathrm{H}_{\mathrm{v}}$ scattering intensity (relative units) at azimuthal angle $\mu=45^{\circ}$ is plotted as a function of the ratio $\Theta_{\mathrm{r}}$ of scattering angle $\theta$ to $\theta_{\max }$ at the maximum intensity, for various values of $\sigma_{0}$ with $R_{\mathrm{m}}=5 \mu \mathrm{m}$. One quadrant of the logarithmic $\mathrm{H}_{\mathrm{v}}$ intensity contours is given in Figure $3 \mathrm{~b}$ as functions of $\theta$ and $\mu$, for $\sigma_{0}=$ 0.1 and 0.3 . Here $\times$ denotes the position of maximum intensity. Similarly, the logarithmic $\mathrm{V}_{\nabla}$ intensity contours are given in Figure 4 for $\sigma_{0}=0.1$ and 0.3 , where the surrounding anisotropy $\alpha_{\mathrm{s}}$ is assumed to be $\left(\alpha_{\mathrm{r}}+2 \alpha_{\mathrm{t}}\right) / 3$. If $\alpha_{\mathrm{s}}=$ $\left(\alpha_{\mathrm{r}}+\alpha_{\mathrm{t}}\right) / 2$ is chosen, the patterns show a little weak intensity around zero scattering angle through all the azimuthal angles. Numerical integration by use of eq 6 is made at intervals of $0.04 \mu \mathrm{m}$ for $\mathrm{d} R$. A general feature of the $H_{v}$ and $V_{v}$ patterns is that the higher order maxima of the intensity disappear for $\sigma_{0}>0.2$; the relatively low intensity contours become long lobes and $\theta_{\max }$ decreases with increasing values of $\sigma_{0}$. It can also be noted that the use of the Gaussian function of eq 7 leads to a somewhat sharp decrease of the intensity around the maximum scattering angle and then to a slow decrease at the larger scattering angles, compared with that for the logarithmic one, within the limit of eq 9.

For convenience in comparing the difference between non-truncated and truncated spherulites, the line $\mathrm{D}$ of Figure 5 gives the logarithmic $\mathrm{H}_{\mathrm{v}}$ intensity at $\mu=45^{\circ}$ for the 67 truncated spherulites having $\left\langle\sigma^{2} / \bar{R}^{2}\right\rangle=0.08$. The lines $\mathbf{A}$, $\mathrm{B}$, and $\mathrm{C}$ in Figure 5 are those for the nontruncated spherulites. The first is calculated from eq 7 with $\sigma=1.5 \mu \mathrm{m}$ and $\bar{R}=5 \mu \mathrm{m}$, and the second and the third are obtained from eq 8 with $\sigma_{0}=0.2$ and 0.15 , respectively, as well as $R_{\mathrm{m}}=5 \mu \mathrm{m}$. These curves are adjusted to agree in the intensity at the maxima. Curve $\mathrm{C}$ is obtained using a distribution based on the average radii of truncated spherulites with $\sigma_{0}=$ 0.15 . However, the scattering pattern of curve 


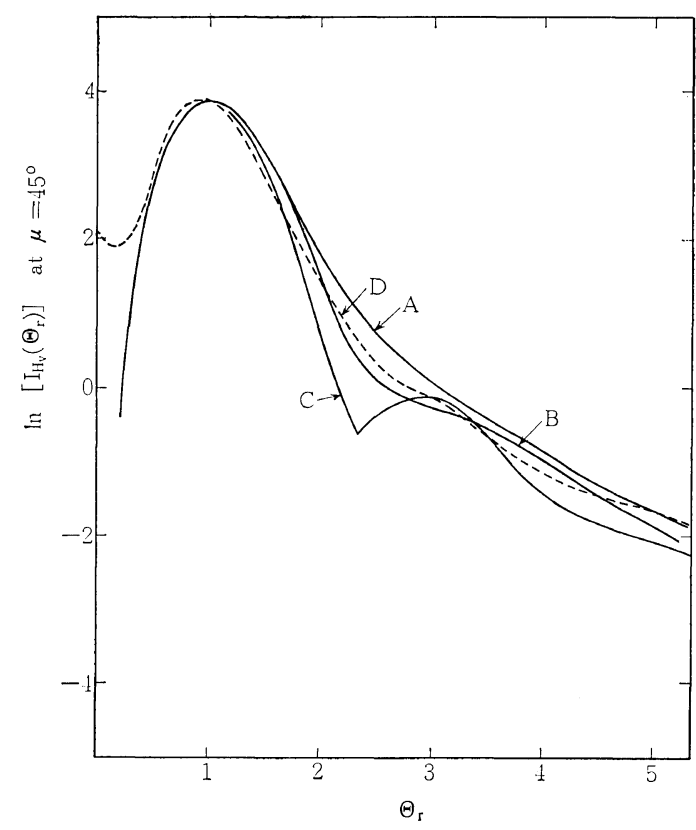

Figure 5. Comparison of the logarithmic $\mathbf{H}_{\mathrm{V}}$ pattern at $\mu=45^{\circ}$ between circular and truncated spherulites as a function of $\Theta_{\mathrm{r}}: \mathrm{A}, \sigma=1.5 ; \bar{R}=5$ $\mu \mathrm{m} ; \mathrm{B}, \sigma_{0}=0.2, R_{\mathrm{m}}=5 \mu \mathrm{m} ; \mathrm{C}, \sigma_{0}=0.15, R_{\mathrm{m}}=5 \mu \mathrm{m}$; $\mathrm{D}$, truncated spherulites of Figure 1 .

$\mathrm{D}$ is found to be close to the curve A $(\sigma=1.5$ $\mu \mathrm{m})$ and the curve $\mathrm{B}\left(\sigma_{0}=0.2\right)$, except in the vicinity of zero scattering angles. The agreement between curves $\mathrm{A}$ and $\mathrm{D}$ is the result of $\langle\sigma\rangle=1.45 \mu \mathrm{m}$ for the assembly of truncated spherulites. By the use of the distribution function based on the mean radii obtained from an assembly of truncated spherulites, an intensity pattern containing all the shape disorder cannot be obtained. This is due to the fact that the distribution function represented by the average radii has a defect: it is truncated at the two ends of the realistic frequency function. An advantage in the use of a distribution function is that a longer calculation, to sum the scattering by individual spherulites as required for the truncated system, can be avoided.

In the solid $\left(\mathrm{H}_{\vee}\right.$ pattern at $\left.\mu=45^{\circ}\right)$ and broken $\left(V_{\nabla}\right.$ one at $\left.\mu=0^{\circ}\right)$ curves of Figure 6 , the shift effect of $\theta_{\max }$ is obtained as a function of $\sigma_{0}$, where the parameters used are the same as those for Figures 3 and 4, respectively. The slight deviation of shift angle between the two inten-

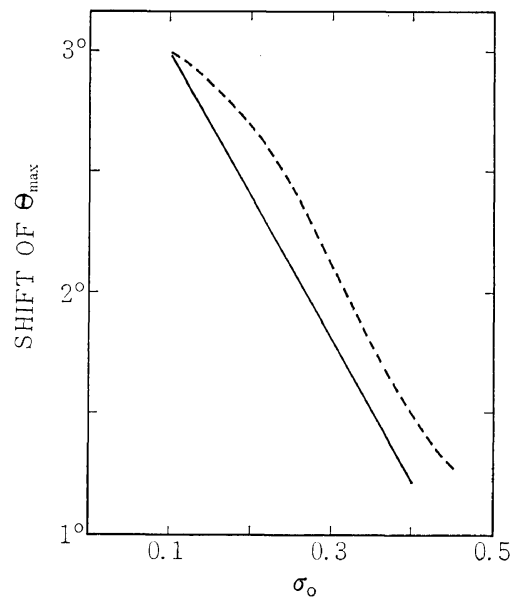

Figure 6. Shift effect of position of $\theta_{\max }$ as a function of $\sigma_{0}$. Solid and broken lines represent the shifts for the $H_{v}$ pattern at $\mu=45^{\circ}$ and the $\mathrm{V}_{\nabla}$ pattern at $\mu=0^{\circ}$, respectively.

sities appears due to intervention of the surrounding anisotropy in the $V_{v}$ function and to the difference of the azimuthal angle leading the maximum intensity. The shift effect is identical to the effect stated previously, ${ }^{6}$ that the larger spherulite sizes are more heavily weighted in the scattering function than the smaller ones. This effect, resulting from an external size distribution, is distinguished from the effect of the internal distribution arising from different truncation parameters. ${ }^{6}$ Thus, it is seen that the shift of the position of $\theta_{\max }$ for an assembly of truncated spherulites is the result of the combination of two effects.

We are now interested to see the relation between the shape of the spherulite and the position of the nucleus. It is known that a truncated spherulite having the shape of a square, triangle, or semicircle does not have any appreciable excess $H_{v}$ intensity at small scattering angles. ${ }^{5}$ We confirm that no change of $H_{\nabla}$ intensity near $\theta=0^{\circ}$ for the above three spherulites can be obtained by the shift of the position of the nucleus within 10\% from its original position, though an increase of the truncation parameter and of the intensity at larger angles is recognized. It is evident that the excess $\mathbf{H}_{\nabla}$ scattering at small angles does not principally result from the variation of the truncation 
parameter, so that this parameter is not sufficient to characterize this feature of the scattering pattern. We next introduce asymmetry for a spherulite having a rectangular shape, letting one side first become $10 \%$ greater than the length of the other; then the ratio of intensity at the maximum to that at zero scattering angle and the truncation parameter increase about $10^{3}$ and 1.2 , respectively. When one side becomes twice as long as the other, the above values increase to about $10^{5}$ and 8 , respectively. Kawai, et al.,$^{10,15}$ have also recognized the excess $H_{\nabla}$ intensity near $\theta=0^{\circ}$, using sheaflike and eccentric circular spherulites as asymmetric objects. Thus, for an internally ordered spherulite, this is the principal origin of the excess $\mathrm{H}_{\nabla}$ scattering at $\theta=0^{\circ}$. We see the shift effect of the nucleus position for the latter spherulite. The original position is set at the center of the circumscribed circle of radius $R$. The $\mathrm{H}_{v}$ scattering pattern at $\mu=45^{\circ}$ of a single rectangular

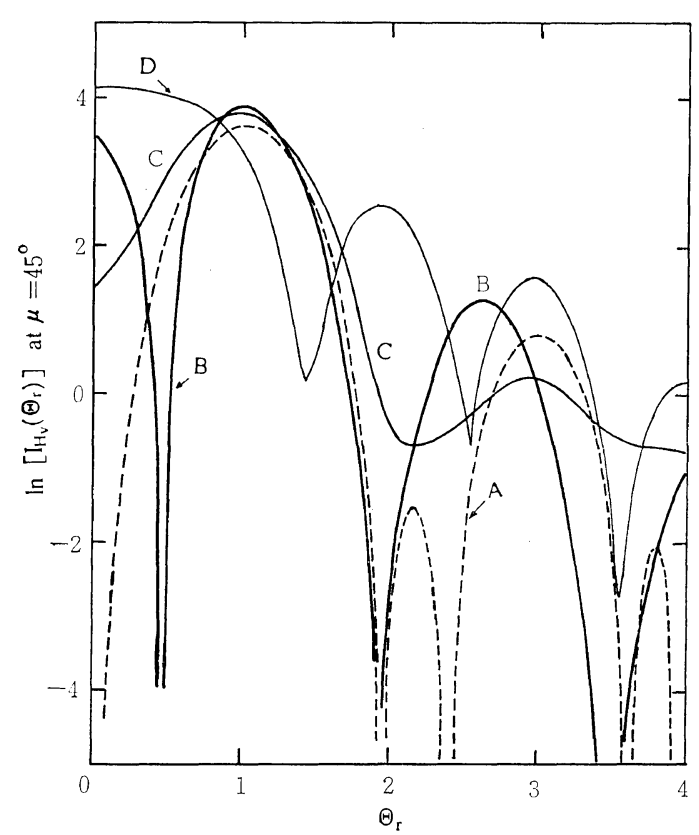

Figure 7. Effect on the logarithmic $H_{v}$ pattern at $\mu=45^{\circ}$ of the transfer of nucleus position of a single truncated spherulite. Broken and solid lines represent the intensities of a square and a rectangular spherulite having length ratio $2: 1$, respectively: A, B, no shift; C, shift to the minor axis direction by $R / 4 ; \mathrm{D}$, shift to the major axis by $R / 2$. spherulite having the length ratio $2: 1$ is expressed by the solid lines of Figure 7 . The broken line represents the pattern for a square spherulite taken as a standard. The marks A, $B, C$, and D represent transfers of the nucleus: $A$ and $B$, no shift; $C$, shift to the minor axis direction by $R / 4 ; \mathrm{D}$, shift to the major axis by $R / 2$. The variance of the truncation parameter is $0.011(\mathrm{~A}), 0.085(\mathrm{~B}), 0.233(\mathrm{C})$, and $0.245(\mathrm{D})$. It is seen that the shift effect of the nucleus for the $\mathrm{H}_{v}$ scattering at $\theta=0^{\circ}$ is considerably greater for $C$ and $D$. On the other hand, such behavior does not become so appreciable for a symmetrical spherulite, even if a truncation parameter exceeds unity for some of such spherulites. Thus, it appears that for the case of polygonal spherulites observed experimentally, for which the truncation parameter is less than 0.13 at most, ${ }^{6}$ the variance of the $\mathrm{H}_{\mathrm{v}}$ pattern caused by the shift of nucleus positions by several percent may not be great. It is generally noted that the internal size distribution for a single truncated spherulite is multi-modal, while the distribution for the assembly of such spherulites may be approximated as mono-modal. The amount of the excess $H_{v}$ scattering is considerably dependent on the shape of the spherulite, and the excess intensity at larger scattering angles comes mainly from the size distribution.

We deleted the interference effect here, but the previous conclusion ${ }^{16}$ was that this produces a speckle pattern without changing the general appearance of the intensity pattern.

\section{EFFECT OF DISORDER}

We shall now consider the effect of internal disorder in the assembly of truncated spherulites, including the size distribution effect. In the previous work ${ }^{3}$ it was not readily possible to consider the combined effects of truncation and disorder. The manner of calculation is the same $^{3}$ except for the truncation effect. The computing time for the case of $M=100$, which is the number of lattice cells along the radius of the spherulite, is about a half hour per single spherulite, so from the limitation of computing time we choose only 20 spherulites in the middle area in Figure 1 as a representative assembly of spherulites. This size distribution is repre- 
Light Scattering by Computer Simulation

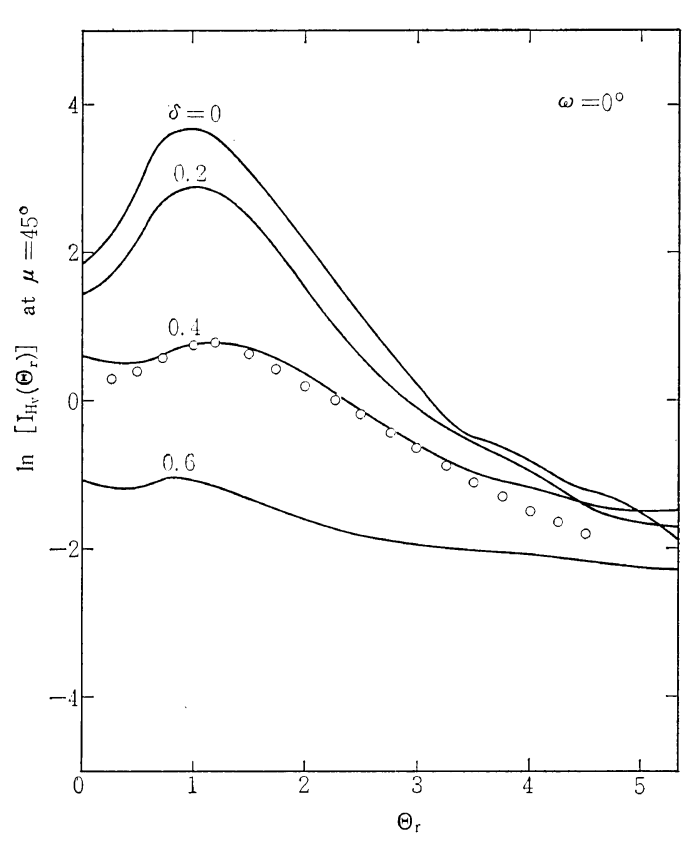

Figure 8. Disorder effect of the logarithmic $\mathbf{H}_{\nabla}$ intensity at $\mu=45^{\circ}$ for 20 truncated spherulites as functions of $\delta$ and $\Theta_{\mathrm{r}}$. Circles are reproduced from experimental values of slowly cooled medium density polyethylene. ${ }^{17}$

sented by $\sigma_{0} \simeq 0.3$. We divide the radius into 100 lattice layers only for the spherulite having maximum radius, keeping the layer thickness the same size for all the other spherulites. Thus our cell size is over $500 \AA$. It should be noted that the intensity pattern depends considerably upon the values of a particular set of random numbers, so the calculation should be made several times at least to average out this statistical variation. In Figure 8 the logarithmic $\mathrm{H}_{\mathrm{v}}$ intensity patterns at $\mu=45^{\circ}$ for several values of the disorder parameter $\delta$ are plotted against scattering angles with a return rate $C_{1}=0.01$ and $\omega=0^{\circ}$ as defined in ref. 3. The intensities are taken by averaging results for 5 sets of random numbers. For comparison, circles which represent experimental values for slowly cooled medium density polyethylene obtained by Hashimoto, et $a l .{ }^{17}$ are included. The maximum peak height is adjusted to the theoretical one for $\delta=0.4$. This choice is rather arbitrary, since the suitable value of $\delta$ varies as $M^{-1 / 2},{ }^{3}$ so the value of $\delta$ for the case of considering the trun- cation effect must be different from the previous one. $^{3}$ It is seen that the agreement between the experimental and theoretical $(\delta=0.4)$ patterns is rather good. If the intensity patterns are modified by experimental conditions, for most cases one may explain them roughly by choosing the appropriate values of $\sigma_{0}$ and $\delta$. One should also recognize the restriction of our calculation, that the disorders of the optic axis in the radial and tangential directions are not actually equal, and the effect for the disorder of twist angle $\omega$ is pending, though special cases for a single spherulite are done and are recognized by a small decrease of the original intensity. ${ }^{3}$ However, we believe that the effect of the disorder of twist angle should be examined for three dimensional spherulites, which would require prohibitive computing time. Finally, it is noted that the relation between the surrounding anisotropy arising from the amorphous parts and the disorder parameter is ambiguous in our model. It must be true that the disorder increases at the cells in the boundary regions among neighbor spherulites, although we treated every cell as unique. The extension of our model for the lattice in three dimensions would be desirable if a faster computer becomes available.

\section{CONCLUSION}

From the above discussion, the conclusion is that the $\mathbf{H}_{\nabla}$ light scattering intensity obtained by summing individual amplitudes from an assembly of truncated spherulites generated by a computer simulation does not agree with that obtained by the use of a distribution function based upon the mean radii obtained from the same spherulite group. However, if the distribution function estimated from the above truncated group is used, a rather good agreement between both patterns can be achieved. The scattering intensity of higher order maxima is found to vanish for $\sigma_{0}>0.2$. The excess $\mathrm{H}_{\mathrm{V}}$ intensity from the truncated spherulites near zero scattering depends mainly upon the shape of the spherulites and at greater scattering angles depends on the size distribution, if the disorder effect is disregarded.

Acknowledgement. The authors wish to thank Dr. A. Misra of Monsanto Company, Springfield 


\section{T. ISHIKAwA and R.S. STEIN}

Mass. who permitted us to extend his work. We also appreciate the helpful comments of Prof. R. E. Prud'homme of Laval University, Quebec, Canada and acknowledge the support of the National Science Foundation and the Army Research Office (Durham).

\section{REFERENCES}

1. R. S. Stein and M. B. Rhodes, J. Appl. Phys., 31, 1873 (1960).

2. R.S. Stein, "Optical Studies of The Morphology of Polymer Films," in "Structure and Properties of Polymer Films," R. W. Lenz and R. S. Stein, Ed., Plenum Publ., New York, N.Y., 1973, p 1. A number of papers are cited in References.

3. D. Y. Yoon and R. S. Stein, J. Polym. Sci. Polym. Phys. Ed., 12, 763 (1974).

4. R. S. Stein, M. B. Rhodes, P. R. Wilson, and S. N. Stidham, Pure Appl. Chem., 4, 219 (1962), and ONR. Technical Report No. 36, Department of Chemistry, University of Massachusetts, Amherst, Mass., 1961.

5. R. S. Stein and C. Picot, J. Polym. Sci., Part A-2, 8, 2127 (1970).

6. R.E. Prud'homme and R. S. Stein, J. Polym. Sci. Polym. Phys. Ed., 11, 1683 (1973).
7. R. S. Stein and W. Chu, J. Polym. Sci., Part A-2, 8, 1137 (1970).

8. R. S. Stein and P. R. Wilson, J. Appl. Phys., 33, 1914 (1962).

9. D. Y. Yoon and R. S. Stein, J. Polym. Sci. Polym. Phys. Ed., 12, 735 (1974).

10. M. Motegi, T. Oda, M. Moritani, and $\mathrm{H}$. Kawai, Polymer J., 1, 209 (1970).

11. S. Clough, J. J. van Aartsen, and R. S. Stein, J. Appl. Phys., 36, 3072 (1965).

12. M. Kerker, "The Scattering of Light and Other Electromagnetic Radiation," Academic Press, New York, N.Y., 1969.

13. Our program is used to make a slight modification in the program of A. Misra, Ph. D. thesis, University of Massachusetts, Amherst, Mass., 1974.

14. R. S. Moore, J. Polym. Sci., Part A-2, 3, 4093 (1965).

15. S. Tatematsu, N. Hayashi, S. Nomura, and H. Kawai, Polymer J., 3, 488 (1972).

16. R.E. Prud'homme and R. S. Stein, J. Polym. Sci. Polym. Phys. Ed., 11, 1357 (1973).

17. T. Hashimoto, R. E. Prud'homme, and R.S. Stein, J. Polym. Sci. Polym. Phys. Ed., 11, 709 (1973). 